\title{
Correction to: Prediction for pathological and immunohistochemical characteristics of triple-negative invasive breast carcinomas: the performance comparison between quantitative and qualitative sonographic feature analysis
}

\author{
Jia-wei $\mathrm{Li}^{1,2} \cdot$ Yu-cheng $\mathrm{Cao}^{3} \cdot$ Zhi-jin Zhao $^{1,2} \cdot$ Zhao-ting Shi $^{1,2} \cdot$ Xiao-qian Duan $^{3} \cdot$ Cai Chang $^{1,2} \cdot$ Jian-gang Chen ${ }^{3}$
}

Published online: 29 October 2021

(c) European Society of Radiology 2021

Correction to: European Radiology

https://doi.org/10.1007/s00330-021-08224-x

The original version of this article, published on 14 September 2021, unfortunately contained a mistake. The following correction has therefore been made in the original: The information that Jia-wei Li, Yu-cheng Cao, and Zhi-jin Zhao contributed equally and that Jian-gang Chen is the first corresponding author and Cai Chang is the second corresponding author was missing. The original article has been corrected.

Publisher's note Springer Nature remains neutral with regard to jurisdictional claims in published maps and institutional affiliations.

The original article can be found online at https://doi.org/10.1007/ s00330-021-08224-x.

Cai Chang

changc61@163.com

$\triangle$ Jian-gang Chen

jgchen@cee.ecnu.edu.cn

1 Department of Medical Ultrasound, Fudan University Shanghai Cancer Center, No 270, Dong' an Road, Xuhui District, Shanghai 200032, China

2 Department of Oncology, Shanghai Medical College, Fudan University, No 270, Dong' an Road, Xuhui District, Shanghai 200032, China

3 Shanghai Key Laboratory of Multidimensional Information Processing, East China Normal University, \#500 Dongchuan Rd., Shanghai 200241, China 